\title{
Collecting EUV mask images through focus by wavelength tuning
}

\author{
Kenneth A. Goldberg ${ }^{1}$, Iacopo Mochi ${ }^{1}$, Sungmin $\mathrm{Huh}^{2}$ \\ ${ }^{1}$ Center for X-Ray Optics, Lawrence Berkeley National Laboratory, Berkeley, CA 94720 \\ ${ }^{2}$ SEMATECH, 255 Fuller Road, Suite 309, Albany, NY 12203
}

\begin{abstract}
Using an extreme-ultraviolet (EUV) microscope to produce high-quality images of EUV reticles, we have developed a new wavelength tuning method to acquire through-focus data series with a higher level of stability and repeatability than was previously possible. We utilize the chromatic focal-length dependence of a diffractive Fresnel zoneplate objective lens, and while holding the mask sample mechanically still, we tune the wavelength through a narrow range, in small steps. In this paper, we demonstrate the method and discuss the the relative advantages that this data collection technique affords.
\end{abstract}

Keywords: extreme ultraviolet lithography, EUV, actinic mask inspection, reticle, Fresnel zoneplate, imaging.

\section{INTRODUCTION}

Aerial image measurement plays a key role in the development of patterned reticles for each generation of lithography. Using EUV-wavelength, "actinic" microscopy to study the field reflected from EUV masks provides a detailed view of the reflective mask surface, including line and defect properties, and the performance of defect repair strategies. ${ }^{1}$ Aerial images have continuous intensity levels, and are not degraded by the complications of photoresist imaging. Furthermore, the measured aerial image intensity distributions can be used as feedback to improve mask and lithography system modeling methods. ${ }^{2}$ In addition to basic mask research and development, interest in commercial standalone EUV mask inspection tools motivates the development of research prototypes and new ideas.

We operate the SEMATECH Berkeley Actinic Inspection Tool (AIT), a synchrotron-based EUV microscope that uses high-magnification Fresnel zoneplate lenses with various, selectable numerical aperture values to directly image blank or patterned EUV masks. ${ }^{3}$

In the most common modes of data collection, we have observed that image-to-image mechanical motion of the mask and zoneplate limit the accuracy and repeatability of the measurements. A new method of data collection utilizes the special wavelength-sensitive optical properties of Fresnel zoneplate lenses to achieve a high degree of stability. The improvement in imaging quality and repeatability has been a singificant advance for the AIT. Since zoneplate imaging requires narrow-band illumination, and this method is made possible by having a continusouly selectable centralwavelength, the method is well suited to synchrotron sources but is difficult to envision in other circumstances, owing to the lmitations of current EUV source technology.

\section{AIT SYSTEM DESIGN AND BEAM PATH}

The AIT experimental configuration has been described previously. ${ }^{3-5}$ Briefly, the AIT operates on a bending magnet beamline at the Advanced Light Source, Lawrence Berkeley National Laboratory. The beamline's monochromator provides EUV illumination with a tunable wavelength centered near $13.4 \mathrm{~nm}$, and an adjustable yet narrow energy bandwidth that we typically operate at $\lambda / \Delta \lambda$ above 1450 to match the requirements set by the zoneplate, which are discussed below.

The configuration of the AIT's imaging optics and light path is shown schematically in Fig. 1 and described in Table 1. Before 2008, only one zoneplate was available for imaging. Since then, the zoneplate design has been modified to include an array of five, user-selectable zoneplates with different optical properties: magnification values are 680, 907, and $1000 \times$; numerical aperture (NA) values are $0.0625,0.075$, and 0.0875 . These NA values emulate the resolution of a $4 \times$ 
Table 1. AIT imaging parameters.

\begin{tabular}{r|l} 
property & value \\
\hline wavelength & $\lambda_{0}=13.40 \mathrm{~nm}$, tunable \\
\hline illumination bandwidth & $\lambda / \Delta \lambda \approx 1450$ \\
available NA values & $\begin{array}{l}\{0.25,0.30,0.35\} \quad(4 \times) \\
\{0.0625,0.075,0.0875\}\end{array}$ \\
zoneplate focal length(s) & $680,750,1000 \mu \mathrm{m}$ \\
magnification & $680,907,1000 \times$ \\
exposure time & $0.5 \mathrm{~s}$, alignment; \\
$30-60 \mathrm{~s}$, high resolution \\
CCD pixel size, number & $13.5 \mu \mathrm{m}, 2048 \times 2048$ \\
pixel equivalent mask size & $19.8,14.9,13.5 \mathrm{~nm}$ \\
observable mask area & approx. 31 $\mu \mathrm{m}$ at 907× mag \\
high-quality field of view & $\begin{array}{l}\text { approx. 2-8 } \mu \mathrm{m}, \text { dependent } \\
\text { on feature size. } \\
\text { approx. 250 images / 8h }\end{array}$ \\
typical data collection rate & app
\end{tabular}

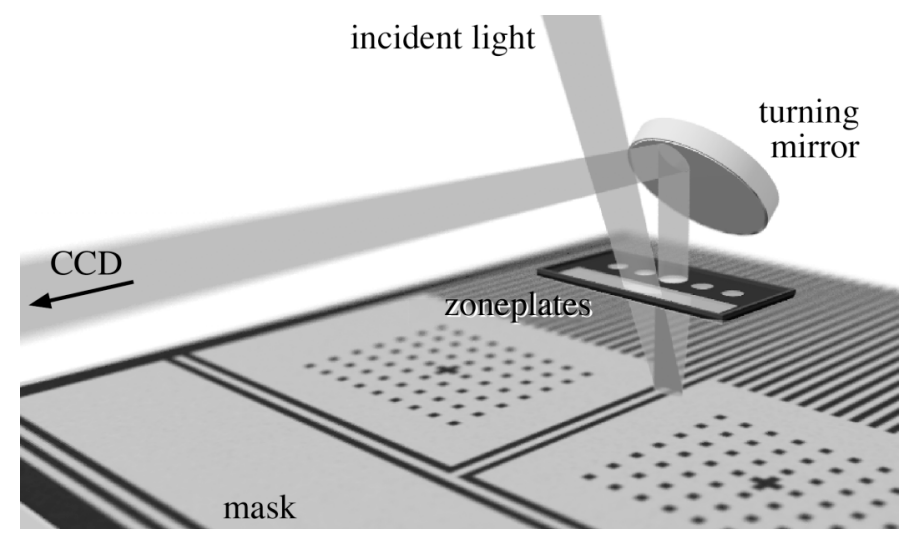

Fig. 1. AIT imaging optics and light path, not to scale. A zoneplate array, held 0.68 to $1.0 \mathrm{~mm}$ above the mask surface, projects a highmagnification image of the illuminated mask surface onto a CCD camera, after reflection form a $45^{\circ}$ multilayer-coated turning mirror.

EUV stepper with NA values of $0.25,0.30$, and 0.35 . The zoneplates are defined in a patterned nickel layer in a siliconnitride membrane. The $6^{\circ}$-incident beam passes through an open region of the absorber and illuminates a mask area approximately $30-\mu \mathrm{m}$-wide. Each zoneplate has a circular pupil, with an off-axis design that creates an angular separation of the various diffraction orders. The first-order focused image is formed with high magnification directly on a backthinned, back-illuminated CCD camera; the zoneplate's undiffracted zeroth-order light is steered below the CCD sensor.

The final turning mirror provides an additional tilt degree of freedom to ensure that the central ray falls close to the vertical center of the CCD camera. The tilt stage gives the mirror $\pm 1^{\circ}$ of adjustment in situ.

\subsection{The challenge of through-focus data collection.}

The most common mode of data collection is to record a series of images "through focus." Despite recent significant advances in the AIT's performance, ${ }^{3}$ the collection of stable and repeatable through-focus data series remained challenging before the adoption of the wavelength-tuning method.

Operating the AIT to collect through focus series, the most significant challenge has been an unavoidable, lateral displacement (by several microns) of the mask and zoneplate. An example of the typical motion in a measurement series is shown in Fig. 2. The combined effects of illumination non-uniformity and the spatially limited, aberration-free sweetspot of the zoneplate lens make it imperative to minimize any lateral displacement during the series. Lateral mask motion changes the local mask illumination and the specific wavefront aberrations of the lens. The illumination nonuniformities arise from contamination in the beamline optical elements, which are now several years old. The effect of the non-uniformities is partially mitigated by scanning the angle of the beamline optics during exposures. ${ }^{3,5}$ Depending on the lens NA and focal length, and the wavefront aberration tolerance, we estimate the sweet spot diameter to be 3-8 $\mu \mathrm{m}$ wide, depending the definition used. ${ }^{6}$ Furthermore, the off-axis imaging geometry creates a tilted focal plane for the lens, so lateral motion in the direction toward the optical axis changes the image defocus as well.
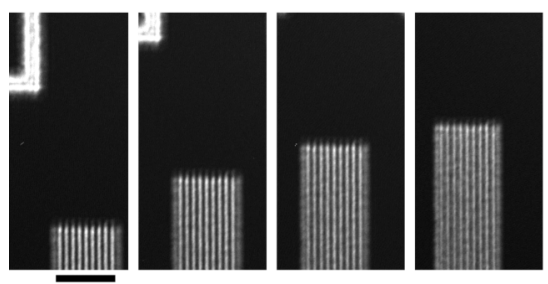

$2 \mu \mathrm{m}$
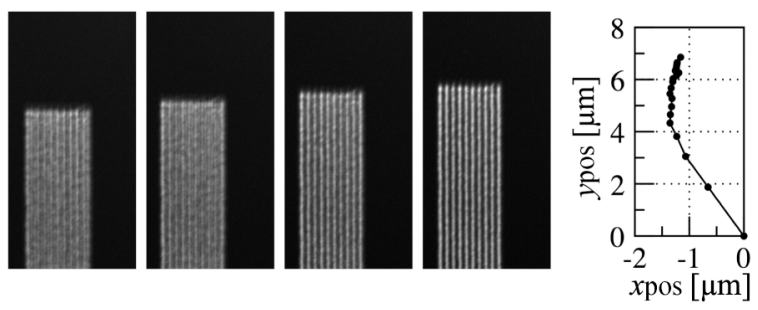

Fig. 2. Lateral motion observed during a typical through-focus series. Lines are 125-nm half-pitch on the mask. Details from the first eight images of the series are shown, from a fixed position on the full CCD image. Nineteen image positions are plotted. The first image position is $(0,0)$. 
We find that longitudinal translation of the mask and zoneplate stages during the steps of the through-focus series is also accompanied by a slow position drift that eventually stops. Stage motion during this relaxation period forces us to impose a 20 second settling time following each $z$ step to achieve stability. With exposure times ranging from 20 to 60 seconds in the highest quality mode, this extra time spent waiting reduces the data collection rate.

\section{WAVELENGTH SCANNING THROUGH-FOCUS SERIES}

We have found that wavelength tuning over a narrow range near the peak of the multilayer band-pass can provide an alternate means of collecting through-focus data without mechanical translation during the series measurement. This solution utilizes the special optical properties of the Fresnel zoneplate lens. A Fresnel lens is a hologram of a simple lens - an electron-beam-patterned binary optical structure of opaque and transparent zones that projects an image of the mask surface into the first diffraction order. The off-axis zoneplate geometry of the AIT zoneplate is shown schematically in Fig. 3.

Diffraction is an inherently wavelength-dependent phenomena. At each point of the lens surface, the local diffraction angle changes with changing wavelength, causing the focal length of the zoneplate lens to vary as well. This effect sets a tight tolerance on the bandwidth of the illuminating light to avoid chromatic image blurring, but it also allows us to tunably control the focal length in a very simple manner: by adjusting the monochromator's wavelength setting. The monochromator's exit slit remains in a fixed position in the beam, while the angle

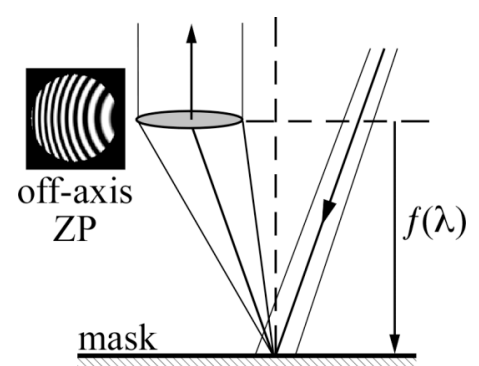

Fig. 3. The geometry of the off-axis zoneplate (ZP) imaging. The focal length $f$ varies inversely with the wavelegnth, $\lambda$, about the design position. of its diffraction grating is adjusted slightly to change the transmitted wavelength.

\subsection{Wavelength dependence of the defocus.}

The relationship between the illumination wavelength and the zoneplate's focal length can be derived in a few steps. We can consider each portion of the zoneplate to be a local grating with a pitch value, $d$, that diffracts light to an angle satisfying the first order grating equation.

$$
\lambda=d \sin (\theta) .
$$

When a zoneplate is designed for wavelength $\lambda_{0}$ with a focal length $f_{0}$, it is easy to show ${ }^{7}$ that it will focus light of a different wavelength $\lambda$ to a focal length $f$, where

$$
f_{0} \lambda_{0}=f \lambda .
$$

This relationship occurs because the lens' zone pattern is fixed, and the sines of the diffraction angles depend linearly on the wavelength. In cases where the illumination wavelength can be adjusted experimentally, we can invert this relationship to manipulate the focal length, $f$,

$$
f(\lambda)=f_{0} \lambda_{0} / \lambda
$$

If the object and lens remain stationary, the relative change in the focal length induces an effective change in the object's longitudinal position through-focus. We can define an effective defocus parameter $\Delta Z_{\text {eff }}$ relative to the original focal length,

$$
\begin{aligned}
\Delta Z_{\text {eff }} & =f(\lambda)-f_{0} \\
& =f_{0}\left(\lambda_{0} / \lambda-1\right) .
\end{aligned}
$$

For small differences, the relationship between wavelength change $\Delta \lambda$, defined as $\Delta \lambda=\lambda-\lambda_{0}$, and the effective position through focus is approximately

$$
\begin{aligned}
\Delta Z_{\text {eff }} & =-f_{0} \Delta \lambda / \lambda \\
& \approx-\left(f_{0} / \lambda_{0}\right) \Delta \lambda, \quad \text { or } \quad \frac{\Delta \lambda}{\lambda_{0}} \approx-\frac{\Delta Z_{\text {eff }}}{f_{0}} .
\end{aligned}
$$




\subsection{The useful range of wavelength tuning.}

Adjusting the wavelength raises several key questions, both experimentally, and for the data's validity. Practically speaking, the amount of defocus required to perform an experimental through-focus series depends on the features sizes of interest. Large features have a greater depth of focus, and require a larger series to study well; yet conversely, small features are frequently of greatest interest in our work, and their depth of focus can be limited to just a few microns.

Using the relationships described in Eqns. (4) and (5), we observe that we can step through the entire useful focus range by tuning the wavelength by a small amount. This relationship is shown in Figure 4. Considering zoneplates with focal lengths 750,1000 , and $1250 \mu \mathrm{m}$, the width of the wavelength range required to perform an $8 \mu \mathrm{m}$ through-focus series is $0.143 \mathrm{~nm}, 0.107 \mathrm{~nm}$, and $0.086 \mathrm{~nm}$ respectively. The longer focal-length lens requires the smallest wavelength change.

An important consideration is the finite multilayer bandwidth of the masks under inspection. A common rule of thumb is that a multi-element EUV optical system has a roughly $2 \%$ bandpass, which at $13.4-\mathrm{nm}$ wavelength, is equivalent to $0.27 \mathrm{~nm}$. Yet a given mask may have a significantly wider wavelength range. Figure 5 illustrates this point with a nearly ideal multilayer mirror illuminated at $6^{\circ}$ form normal incidence. Neglecting the role of other optical elements in the illumination system, the reflectivity full width at half maximum (FWHM) could set a practical working range for through-focus wavelength tuning. Yet the multilayer's bandpass is close to $4.3 \%$ and thus broad enough that it sets no limitation on the technique for our applications.

Furthermore, the optical properties of the absorber materials are not expected to change appreciably over the narrow ranges of the proposed wavelength tuning. Phase masks, however, are a special wavelength-sensitive case and must be evaluated specificially.

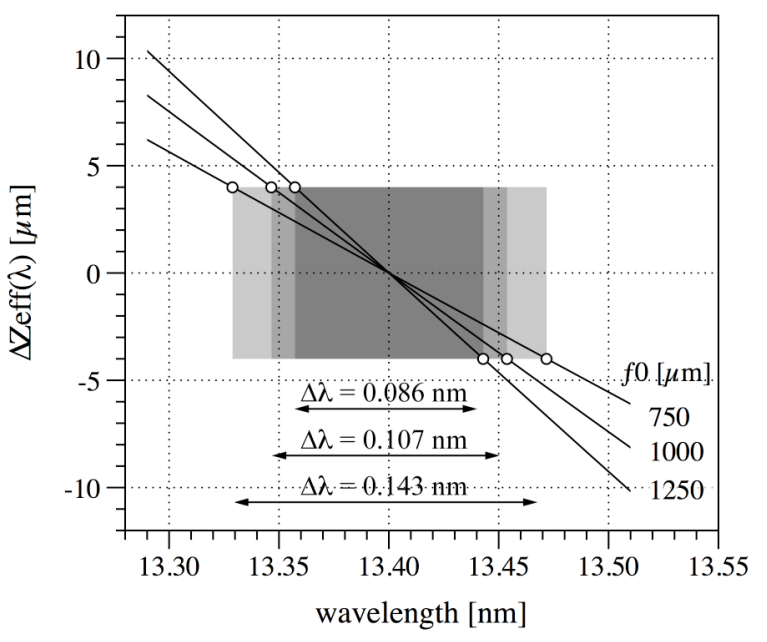

Fig. 4. The effective $z$ position change as a function of changing wavelength, calculated according to Eq. (4) for three different focal lengths: 750, 1000, and $1250 \mu \mathrm{m}$. The shaded rectangular regions show the total wavelength range required to perform $8 \mu \mathrm{m}$ through-focus series - much larger than is usually necessary.

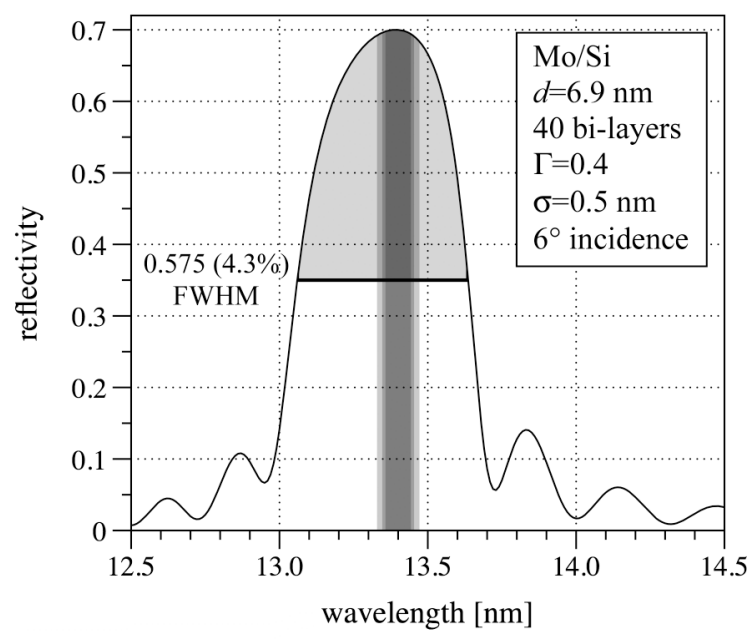

Fig. 5. Reflectivity vs. wavelength curve of an ideal multilayer mask structure with a small amount of layer interdiffusion. The wavelength ranges from Fig. 4 are overlayed here. The realatively broad wavelength bandpass $(0.575-\mathrm{nm}$ FWHM) should not limit the through-focus wavelength tuning, which typically uses a narrower wavelength range.

\subsection{Practical zoneplate bandwidth considerations.}

The same zoneplate optical properties that make the wavelength tuning through focus method possible, also constrain the allowable illumination bandwidth: the reasoning is identical. Each wavelength component within the illumination bandwidth has its own corresponding $\Delta Z_{\text {eff }}$, and contributes to the final image. The significance of the resultant image blurring depends on the bandwidth and on the object feature sizes being studied. In the following discussion all units are given relative to the mask. Conversion to $4 \times$ or $5 \times$ imaging requires division of the mask lateral dimensions by 4 or 5 , and of the longitudinal (focus) dimensions by 16 or 25 , respectively.

Although the AIT has recently demonstrated 70\% image contrast for 88 -nm dense lines (mask units), ${ }^{3}$ more common half-pitch feature sizes of interest fall in the range of 100-200 nm. The total depth of field for the smallest size in this 
range is close to $3 \mu \mathrm{m}$. We commonly operate the monochromator with a spectral bandwidth of $\lambda / \Delta \lambda=1450$. Following Eq. (5), the longitudinal blur at this bandwidth is $0.5 \mu \mathrm{m}$. Therefore, making focal steps at finer increments, whether by wavelength tuning or by $z$ motion, creates a series with overlaping focus information. Should it become necessary to resolve with finer through-focus increments, the bandwidth would have to be reduced or the zoneplate design changed to shorten the focal length.

\section{EXPERIMENTAL DEMONSTRATIONS}

This section presents two experiements to demonstrate the wavelength tuning through-focus data collection method. In one case we recorded several series at different physical mask- $z$ positions and compare the contrast vs. defocus curves. The second example is a comparison of two series recorded with the traditional and new methods respectively showing the quality of the information that can be collected from EUV aerial image measurements.

\subsection{Measurement at three focus positions.}

If the $z$-motion and wavelength tuning methods are interchangeable within the narrow focus range, then multiple through-focus series recorded at different mask $z$ positions should overlap when the various physical and wavelengthdependent displacements are compensated. Figure 6 contains an example of such a measurement, performed using 250-nm dense darkfield lines. Three separate series were recorded using wavelength tuning; between the measurements the mask was displaced in $z$. Figure 6a shows the measured contrast versus the effective $z$ values predicted by wavelength tuning, before the mask $z$ displacements are included. In Fig. 6b, the $z$ displacements are included and the curves overlap. Although the displacents were known approximately, the data itself was used to determine the $z$ displacements more precisely. For each series, the wavelength-tunings' central wavelength values are given in the figure.
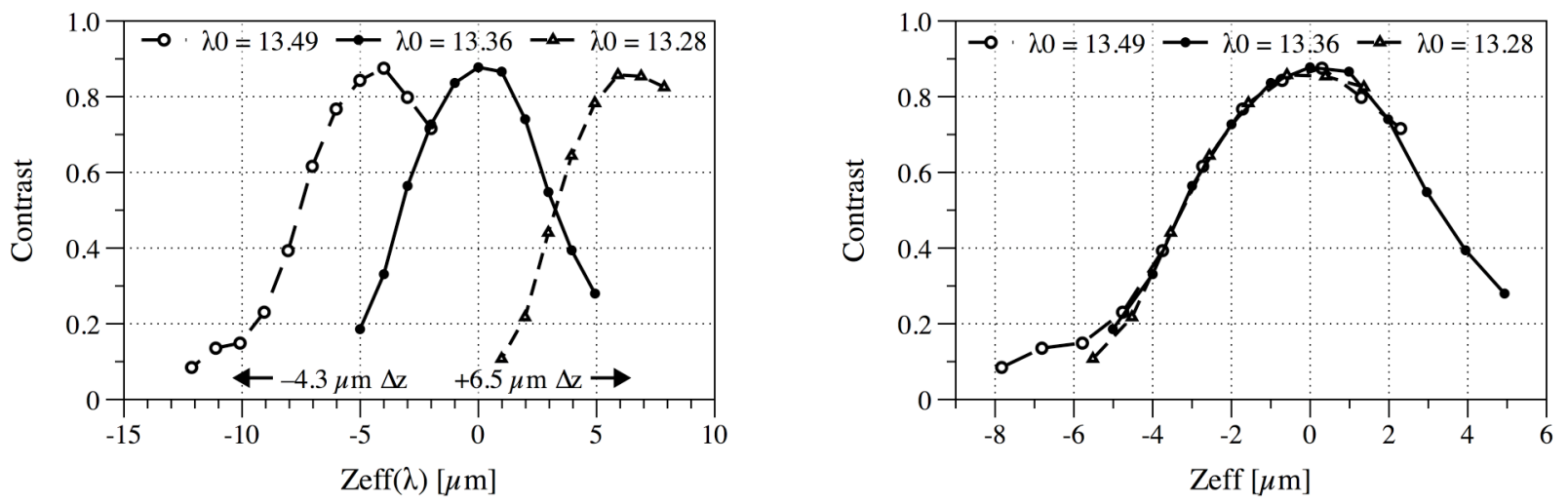

6a. Three through-focus scans made by wavelength tuning at $\mathbf{6 b}$. The same three through-focus scans from $3 \mathbf{a}$ are shown with different $z$ positions. The central wavelength for each scan is $Z_{\text {eff }}$ now including both the wavelength-dependent focal position shown. Here, $Z_{\text {eff }}(\lambda)$ is defined as the wavelengnth-dependent change, and the physical change of the mask position.

focal change, before the physical mask displacement is included.

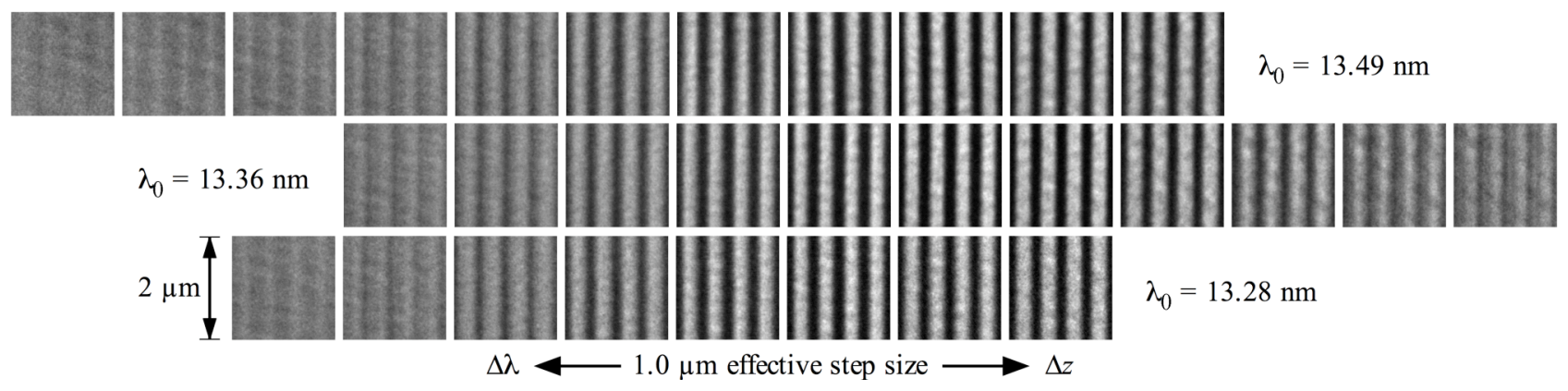

Fig. 7. Aerial image details from the three wavelength-tuning through-focus series (left to right), also shown in Fig. 6. The central wavelength of each series is shown to the side of each. The (estimated) physical mask displacements between the series are shown in Fig. 6a. 


\subsection{Comparing data from $z$ motion and wavelength-tuning through-focus scans.}

Data presented in Fig. 8 shows the kinds of aerial image line measurements that are possible with the AIT using both of the through-focus data collection modes. Two different masks were studied using $125-\mathrm{nm}$ dense lines in both cases. The zoneplate objective's NA value is 0.0875 , equivalent to a 0.35 -NA $4 \times$ stepper. The focal length is $750 \mu \mathrm{m}$, and the magnification ratio is 907 .

The aerial image line parameters were studied using ThroughFocus software. ${ }^{8}$ The calculations of contrast, critical dimension (CD), normalized image log slope (NILS), and line-width roughness (LWR), are based on small regions measuring $0.25 \times 2.00 \mu \mathrm{m}(2 \times 16 \mathrm{CD})$. NILS and LWR are calculated at the 1:1 intensity threshold determined separately for each series in the best-focus image, determined by peak contrast. Unless otherwise marked, all units shown are given in mask scale. In each data series, the presence of noise is unavoidable. In addition, small but finite field-dependent lens aberrations affect the line shapes in subtle ways. Separately, each mask we study has a fixed pattern of observable roughness that may originate as multilayer phase roughness. ${ }^{9}$

Mask A

Through-focus $z$ series, Oct. 2008
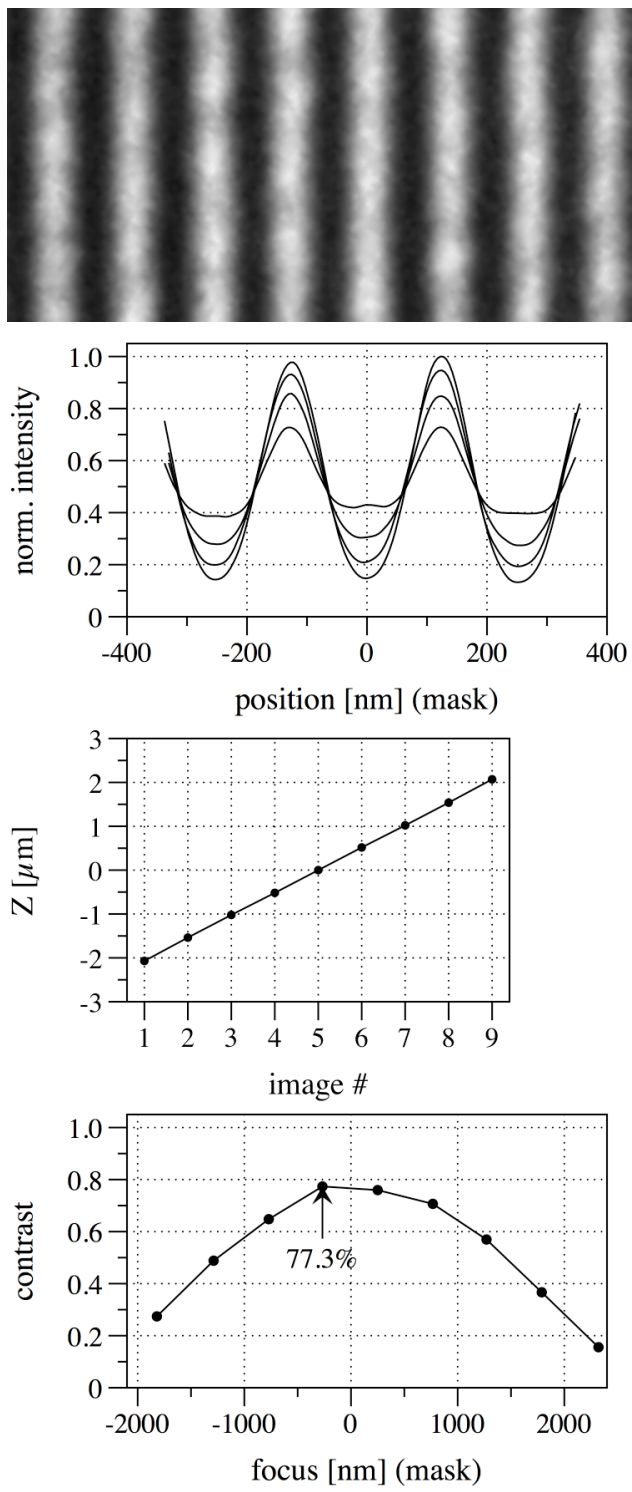

Mask B

Through-focus $\lambda$-series, Dec. 2008
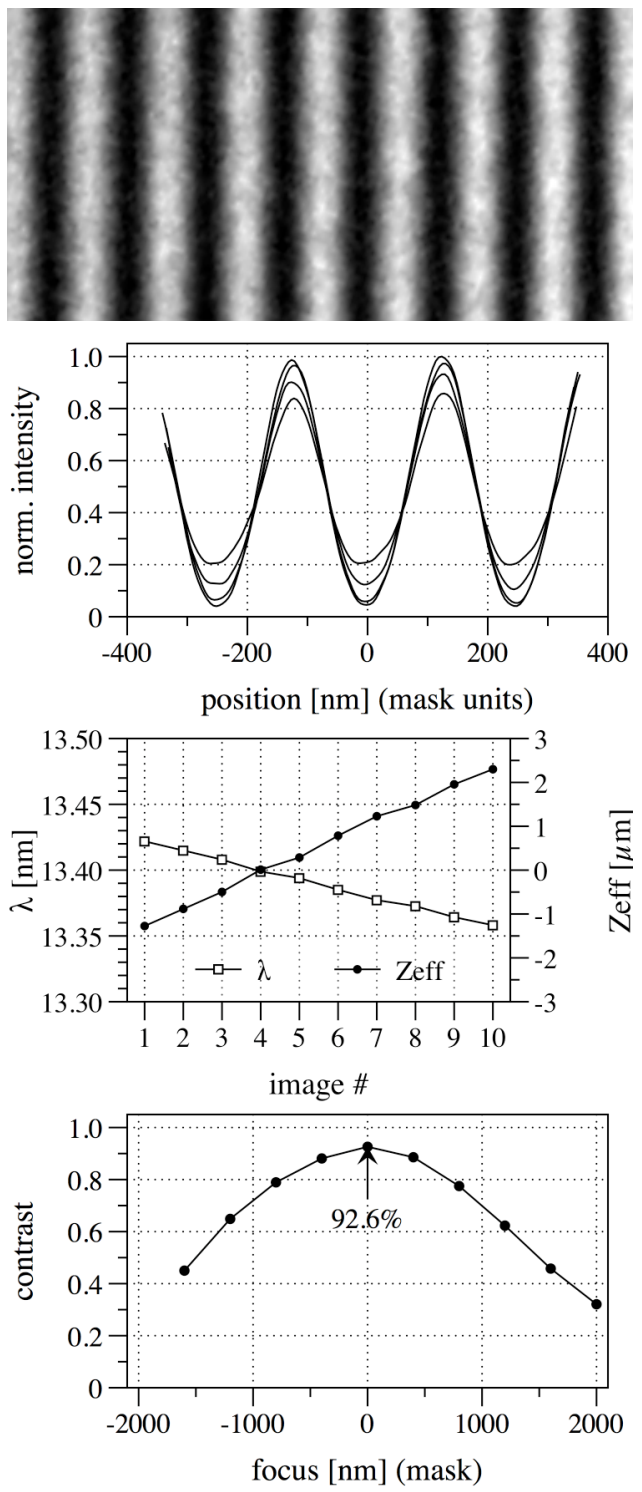

8a. Aerial images of 1:1 dense lines at best focus. The regions shown are $2 \times 1 \mu \mathrm{m}$ wide, and the lines are $125 \mathrm{~nm}$ half-pitch, on the mask ( $31 \mathrm{~nm}$ in $4 \times$, and $25 \mathrm{~nm}$ in $5 \times$ wafer scale.)

8b. Intensity profiles throughfocus in four steps, normalized to the peak in the peak-contrast image. The effective $z$ steps sizes are $0.5 \mu \mathrm{m}$ and $0.4 \mu \mathrm{m}$, respectively. Conversion to wafer units requires division by 4 or 5 .

8c. Through-focus steps, performed with physical $z$ mo$\bar{\Xi}$ tion (left), and using waveミ length tuning to control defocus (right). For wavelength tuning, the effective defocus $\left(Z_{\text {eff }}\right)$ is also shown.

8d. Contrast through focus. The peak contrast difference is attributable to the specific properties of the two different masks. Line properties are calculated in a $0.25 \times 2.00$ $\mu \mathrm{m}$ region, along the direction of the lines. 
Mask A

Through-focus $z$ series, Oct. 2008
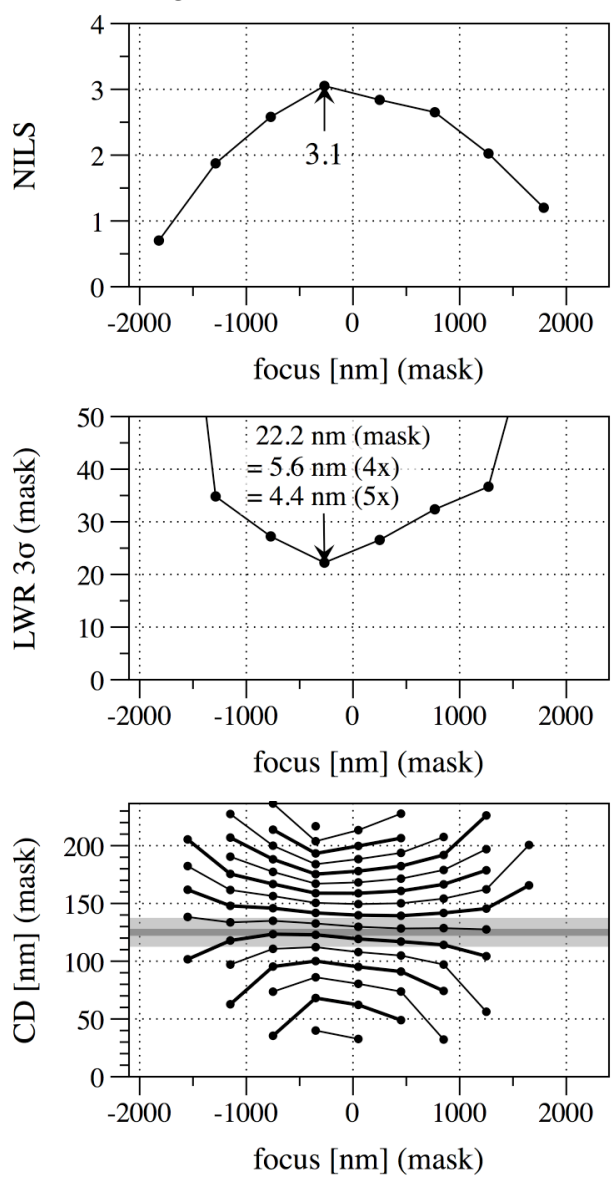

Mask B,

Through-focus $\lambda$-series, Dec. 2008
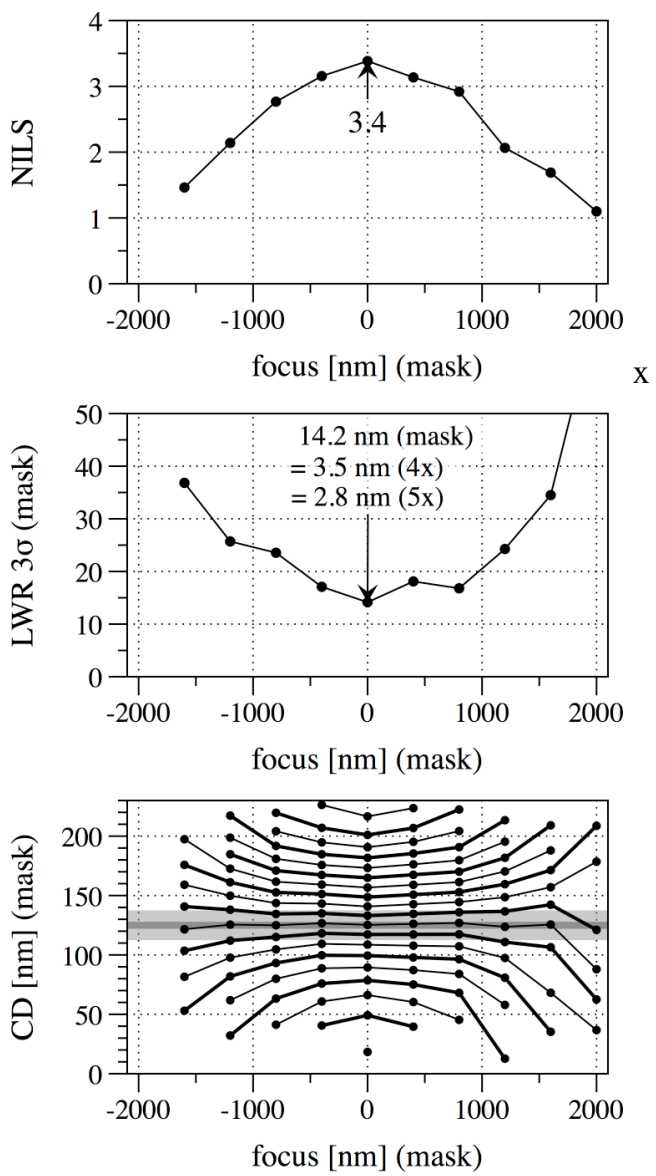

8g. CD through focus at different normalized intensity threshold levels, spaced by 0.05 . The gray bar represents $\pm 10 \%$ CD change form the 125 -nm programmed value. 8e. NILS through focus. Normalized image log slope calculated at the 1:1 linespace intensity threshold determined at best focus (peak contrast) in each series.

8f. LWR $3 \sigma$ through focus. Line width roughness is calculated at the 1:1 linespace intensity threshold. Equivalent $4 \times$ and $5 \times$ sizes are also shown for the minimum measured value.

\section{SUMMARY}

Aerial image mask measurements from the AIT provide a detailed look at the mask surface revealing the properties of lines and defects, the success of repair strategies, and the accuracy of modeling. Bossung series can be extracted from a relatively small number of images collected through-focus.

The imaging quality from the AIT has improved significantly over time as a result of improved zoneplate designs, ${ }^{3,5}$ fine system alignment with aberration feedback, ${ }^{6,10}$ and improved data collection methods. Despite these advances the AIT suffers from a step-wise lateral position drift during through-focus series, as a result of its unusual stage design. This motion degrades the quality of the images and of the series as a whole.

We have utilized the wavelength-dependent properties of zoneplate lenses to develop an improved method of data collection through focus that does not involve any motion of the mask or zoneplate once initial alignment has been performed. Data collection speed and stability have both increased. The simple relationship between the illumination wavelength and the focal length of a zoneplate objective allows the defocus to be varied in arbitrary steps, without sacrificing mechnaical stability or position. Since the adoption of the wavelength-tuning method, it has become the AIT's dominant mode of data collection, resulting in improved repeatablity and self-consistency of the data.

As preseted here, the method is well suited only for narrow-band tunable light sources. However, in those cases where the sample's optical properties do not vary across a wavelength range of interest, the wavelength tuning method could be effective-where appropriate, it could easily be adopted on other EUV, soft-x-ray, and x-ray miroscopes that require data collection in different focal planes. 


\section{ACKNOWLEDGMENTS}

The authors gratefully acknowledge Nathan Smith, Charles D. Kemp, and Senajith Rekawa who provided dedicated engineering and technical assistance that made all of these measurements possible; contributing scientists Eric Gullikson, Patrick Naulleau, and Anton Barty for their roles in the development and use of the AIT; and Hakseung Han, Pei-Yang Yan, Ted Liang, Bruno LaFontaine, Obert Wood, and Stefan Wurm for their continued guidance and support. Robert Gunion, Paul Denham, Ron Tackaberry, Hanjing Han, Kenneth Woolf, and Jeffrey Gamsby provided expert assistance with software and hardware electronics instrumentation. Masks for this work were provided by Intel.

This work was performed under the auspices of the U.S. Department of Energy by University of California Lawrence Berkeley National Laboratory, and was funded by SEMATECH. Research was conducted by scientists from the Materials Sciences Division of Lawrence Berkeley National Laboratory at the Advanced Light Source with support from the ALS Division. This work was funded by Sematech, Inc., Contract No. 401832-NY through the U.S. Department of Energy under Contract No. DE-AC02-05CH11231.

\section{REFERENCES}

1. H.-S. Han, W. Cho, K. A. Goldberg, E. M. Gullikson, C.-U. Jeon, S. Wurm, "Determining the critical size of EUVmask substrate defects," SPIE 6921, 69211Y (2008).

2. C. H. Clifford, S. Wiraatmadja, T. T. Chan, A. R. Neureuther, K. A. Goldberg, I. Mochi, T. Liang, "Comparison of fast 3D simulation and actinic inspection for EUV masks with buried defects," these proceedings.

3. K. A. Goldberg, I. Mochi, P. P. Naulleau, H.-S. Han, S. Huh, "Benchmarking EUV mask inspection beyond 0.25 NA," SPIE 7122, 71222E-1 (2008).

4. K. A. Goldberg, P. Naulleau, I. Mochi, E. H. Anderson, S. B. Rekawa, C. D. Kemp, and R. F. Gunion, H.-S. Han, S. Huh, "Actinic extreme ultraviolet mask inspection beyond 0.25 numerical aperture," J. Vac. Sci. \& Technol. B 26 (6), 2220-4 (2008).

5. K. A. Goldberg, P. P. Naulleau, A. Barty, S. B. Rekawa, C. D. Kemp, R. F. Gunion, F. Salmassi, E. M. Gullikson, E. H. Anderson, H.-S. Han, "Performance of actinic EUVL mask imaging using a zoneplate microscope," SPIE 6730, 67305E-1-12 (2007).

6. I. Mochi, K. A. Goldberg, S. Huh, "Improving the performance of the AIT with an optimized alignment procedure," these proceedings.

7. D. Attwood, "Soft X-rays and Extreme Ultraviolet Radiation: Principles and Applications" (Cambridge University Press, 1999).

8. ThroughFocus Software, Version 1, Kanayama Consulting, http://ThroughFocus.com, 2008-9.

9. P. Naulleau, K. Goldberg, I. Mochi, G. Zhang, “Mask Effects on LER,” EUVL Symposium 2008, September 2008.

10. I. Mochi, K. A. Goldberg, P. Naulleau, S. Huh, "Achieving Diffraction-limited EUV Aerial Image Microscopy," EUVL Symposium 2008, September 2008. 